\title{
Evaluation of small ncRNAs as a possible epigenetic mechanism mediating the transition from biotrophy to necrotrophy in the life cycle of Phytophthora infestans
}

Juliana Gonzalez-Tobon

Universidad de los Andes Facultad de Ciencias

Alejandra Rodriguez-Jaramillo

Universidad de los Andes Facultad de Ciencias

Laura Milena Forero

Universidad de los Andes Facultad de Ciencias

Laura Natalia González

Universidad de los Andes Facultad de Ciencias

Giovanna Danies

Universidad de los Andes

Silvia Restrepo ( $\nabla$ srestrep@uniandes.edu.co)

Universidad de Los Andes https://orcid.org/0000-0001-9016-1040

Research article

Keywords: Epigenetics, small ncRNAs, microbiology, computational biology, Phytophthora infestans

Posted Date: August 15th, 2019

DOI: https://doi.org/10.21203/rs.2.12907/v1

License: (c) (i) This work is licensed under a Creative Commons Attribution 4.0 International License.

Read Full License 


\section{Abstract}

Background Phytophthora infestans, the causal agent of late blight disease of potato and tomato, is responsible for billions of dollars in losses worldwide each year. This plant pathogen is a hemibiotroph, meaning that in the early stages of infection, it feeds on living host tissue, and later in the infection cycle, it kills the host. Even though the transcription dynamics of this transition are well characterized, information on the role of small noncoding RNAs (ncRNAs) during this process is still being obtained. Furthermore, a methodological pipeline useful for searching and analyzing small ncRNAs in this pathogen is needed. Results Using the proposed pipeline, a set of 146 small ncRNAs were found to be significantly differentially expressed among the analyzed samples. From these, 100 small ncRNAs were successfully annotated to specific genes, some of them encoding effector proteins produced by the pathogen during its life cycle. Other molecular regulatory processes, mainly mediated by enzymes and transporters, were also identified. Conclusions The pipeline presented here is an easy-to-use, comprehensive and efficient option for analyzing small ncRNA profiles of P. infestans small RNA-seq data sets. Additionally, the results presented here may further contribute to elucidating the specific molecular and epigenetic mechanisms controlling the life cycle of P. infestans.

\section{Background}

Phytophthora infestans, the causal agent of late blight disease of tomatoes and potatoes, is an oomycete responsible for billions of dollars in losses annually [1]. A successful crop may be destroyed in just a couple of weeks [2]. This plant pathogen is a hemibiotroph, meaning that on the onset of its life cycle, it feeds on living host tissue (biotroph), and later in the infection cycle, it kills the host (necrotroph). So far, it is not well understood how and why this transition occurs. Nevertheless, it has been suggested to be related to proteins, known as effectors, that are secreted by the pathogen into the host cell $[3,4]$.

These effectors promote pathogen invasion by impairing the host immune responses when specific host resistance genes (R genes) are absent [5]. Some effectors are expressed early in the infection cycle, while others are expressed later on [3]. The effectors are secreted based on an "accelerator and brake" model in which a certain set of effector proteins can either slow down (brake) or accelerate necrosis. Additionally, some effectors act on their own, while others act on additional effectors to generate the effect [4]. Therefore, the regulation of their expression involves a coevolutionary arms race between the pathogen and the host. Phytophthora infestans will acquire effectors for which the host lacks the corresponding $\mathrm{R}$ genes and, conversely, the plant will acquire the $\mathrm{R}$ genes to be able to defend itself from the pathogen.

The gain and loss of effector proteins may occur by long-term stable changes in the effector genes or by much faster and reversible mechanisms. The first possibility is usually accomplished by gain-of-virulence (g-o-v) changes in the gene sequence. These variations usually include pseudogenization or loss of the gene by conventional mutations [5, 6]. Furthermore, it has been hypothesized that g-o-v changes can occur without any alteration of the gene sequence, in other words, epigenetically $[5,6]$. This enables a powerful and versatile reversible control strategy since the coding sequence of the effector genes would 
not be altered and thus the genes can be re-expressed. This allows the pathogen to maintain the genes instead of losing them. Moreover, it would allow the pathogen to activate the expression of the genes much faster, gaining a plethora of opportunities to respond successfully to changing environmental or host constraints.

In $P$. infestans, the epigenetic regulatory mechanism that has been most widely studied is transcriptional gene silencing via small noncoding RNAs (ncRNAs) [6, 7]. Small ncRNAs are known to fulfill different roles, such as developmental regulation, environmental response and host-pathogen interactions [8]. To date, several studies state that species of the genus Phytophthora seem to express small ncRNAs of approximately $19-40 \mathrm{nt}[9,10]$. In the case of $P$. infestans, small ncRNAs seem to be divided into three groups of 21, 25/26 and $32 \mathrm{nt}$. However, in the case of $P$. parasitica, they are divided into two groups of 25-26 nt and $21 \mathrm{nt}$ [8]. Additionally, Vetukuri and collaborators (2012) found endogenous small ncRNAs derived from effector genes.

Research on small ncRNAs of the genus Phytophthora is currently growing and it is believed that small ncRNAs are also used to ensure transposon control in this pathogen that has $75 \%$ of its genome composed of repetitive elements [11]. Most of the small ncRNAs identified by Vetukuri and collaborators (2012) mapped to these repetitive regions overlapping with effector genes [8, 9]. One of the existing hypotheses that seeks to explain how the effector genes are regulated is that ncRNAs may guide transcriptional gene silencing through chromatin modifications [9]. Therefore, the effector genes could be silenced due to heterochromatin extension from the transposon to the effector gene regions $[5,7,9]$. Moreover, Jia and collaborators (2017) found that the genes with which P. parasitica small ncRNAs overlap are 40\% RXLR effector genes, 50\% CRN effector genes and some elicitor genes, all well known for their involvement in host-pathogen interactions. These relationships have also been noted for specific types of small ncRNAs such as micro RNAs and transfer RNAs derived small ncRNAs $[12,13,14]$.

Interestingly, studies specifically analyzing the transition from biotrophy to necrotrophy as it relates to epigenetics are not common. The published studies usually focus on specific small ncRNA types as well as their evolutionary traces in the genome [15]. One of the reasons for this may be the lack of clear bioinformatic pipelines that can analyze this type of data. Existing pipelines for the analysis of small ncRNAs, e.g., sRNAtoolbox [16], iMir [17], and shortran [18], were developed for animal or plant data specifically. Additionally, even though current studies describe the steps they followed and the programs they used to analyze data in similar ways, an established pipeline that can be modified according to the researchers' needs can be a useful addition to the current knowledge on the matter.

The aim of this study was to evaluate whether small ncRNAs can constitute an epigenetic regulatory mechanism for the transition from biotrophy to necrotrophy in $P$. infestans. Additionally, this phenomenon was used as a model to design a useful methodological pipeline for the analysis of small ncRNA data sets in this plant pathogen. The results may further contribute to the elucidation of the specific molecular and epigenetic mechanisms controlling the life cycle of $P$. infestans. 


\section{Results}

\section{Implementation of a bioinformatic pipeline to analyze small ncRNA data}

After implementing the pipeline and assessing the differentially expressed genes using DESeq2, a set of 146 small ncRNAs were found to be significantly differentially expressed among the four samples. This full set is reported in Table $A 1$ along with the $p$-values and $\log _{2}$ fold changes. $\log _{2}$ fold changes for the upregulated small ncRNAs ranged between $2.8-4.4$, and $\log _{2}$ fold changes for the downregulated small ncRNAs ranged between -2 and -4.25 . A total of 100 small ncRNAs were successfully annotated to specific genes. Six ncRNAs were tRNAs and pseudo tRNAs and one was a 28S rRNA. Thirty-nine snRNAs were associated with putative uncharacterized proteins.

The associated genes were divided in nine categories to which the 100 small ncRNAs were classified into molecular functions or cellular processes. Table 1 summarizes the nine categories, as well as the expression level (up- (+) or down- (-) regulated) of the small ncRNAs across each comparison of data sets. The first three categories included small ncRNAs that were associated with genes that were not protein-coding. Therefore, the RNAs generated from their transcription have their own function. These categories were small nucleolar RNA, spliceosomal RNA, and eukaryotic type signal recognition particle RNA. The remaining categories contained small ncRNAs that mapped to protein-coding genes. Three of the categories included effectors of different types: elicitins, crinkler effectors, and RXLR effectors. These categories had a direct and relevant association with the transition from biotrophy to necrotrophy. The remaining three categories (enzymatic processes, transporters and transmembrane proteins, and DNA binding or binding sites) were more general categories. For example, the enzymatic processes category included a wide variety of enzymes, such as glucosidases, proteases, and kinases.

The majority of the annotated small ncRNAs were identified when genes from any of the three infection time points $(24,48$, and $72 \mathrm{hpi})$ were compared to genes expressed in mycelia grown in culture media. Finally, a principal component analysis (PCA) between the four individual data sets ( $24 \mathrm{hpi}, 48 \mathrm{hpi}, 72 \mathrm{hpi}$, and mycelia grown in culture media) is shown in Figure 2. As expected, the mycelia grown in media differed considerably from any of the other data sets than did any of the other data sets between each other. Interestingly, data from $48 \mathrm{hpi}$ and $72 \mathrm{hpi}$ were much more similar between each other than either of them were with the 24 hpi data set.

\section{Generation of qRT-PCR profiles for gene expression validation}

A qRT-PCR profile was obtained for genes associated with 10 small ncRNAs that were predicted to be significantly differentially expressed among the different stages of the infection cycle (biotrophy, hemibiotrophy and necrotrophy) (Table 2). The expression of these genes at each of the three stages of the infection cycle was compared to that of the same genes when $P$. infestans was grown in pea agar. 
The specific levels of up- and downregulation are presented in Figure 1 of Additional File 2. Furthermore, these results are summarized in Figure 3.

As shown in Figure 3, three effector genes were tested. The first, RXLR encoding Avr2 family secreted RxLR effector, was slightly upregulated during biotrophy and then was downregulated during both the transition from biotrophy to necrotrophy and during necrotrophy, which was much more evident during necrotrophy. Two elicitin genes, INF1 and INF6, were also evaluated. Both were downregulated at different magnitudes during all stages of the infection when compared to the mycelia grown in vitro.

On the other hand, three genes associated with enzymatic processes were tested. First, the gene Sporkinase, which encodes a sporangia-induced phosphatidyl inositol kinase (Figure 3), was downregulated in all stages when compared to the in vitro mycelia. Moreover, a gene encoding a peptidylprolyl cis-trans isomerase that showed slight overexpression during biotrophy, minor repression during the transition phase and substantial overexpression again during necrotrophy was tested (Figure 3). Finally, the gene SporeSpecific, which encodes a spore-specific nuclear LIM factor, was evaluated. This gene was upregulated in all stages of the infection when compared to the in vitro mycelia (Figure 3). Last, a gene encoding a Berberine-like protein that seems to have both enzymatic and DNA binding properties was tested. The results showed that this gene was slightly upregulated in the biotrophic stage and then became downregulated during hemibiotrophy and necrotrophy (Figure 3).

Finally, three genes classified as transporters and proteins with transmembrane properties were analyzed. Two of these genes encoded an ATP-binding cassette superfamily ( $A B C)$ transporter. ABC transporter \#1 showed downregulation of approximately $-10 \log _{2} \mathrm{FC}$ during biotrophy, almost null overexpression during the transition and overexpression during necrotrophy. Surprisingly, $A B C$ transporter \#2 did not present any up- or downregulation during any of the stages when compared to the in vitro mycelia. Similarly, the gene encoding a haustorium-specific membrane protein was not differentially expressed during any of the infection stages when compared to the in vitro mycelia (Figure 3).

\section{Discussion}

The present study suggests that small ncRNAs seem to be correlated with some effector proteins expressed in $P$. infestans during the hemibiotrophic life cycle as well as with other molecular regulatory processes. Most likely, small ncRNAs can be involved in one of the regulatory mechanisms mediating the infection processes of $P$. infestans in $S$. tuberosum. Moreover, a useful bioinformatic pipeline was presented and implemented for the analysis of small RNA-seq data sets in P. infestans.

One of the most relevant trends observed in the results obtained bioinformatically was that the small ncRNAs were associated with effector-like proteins. To start, crinkler and crinkler-like proteins, which are well-known effectors associated with the necrotrophic phase in $P$. infestans, were found to be downregulated at the later stages of the infection cycle (48 hpi and $72 \mathrm{hpi}$ ) when compared to in vitro mycelia. Therefore, if these "silencing" small ncRNAs are not expressed at later stages of the cycle, the 
expression of their corresponding genes will not be inhibited, and thus, the production of the associated proteins will be enabled. Since crinkler proteins are mostly associated with the necrotrophic phenotype observed in hemibiotrophic pathogens, this scenario is expected and provides evidence of the association of small ncRNAs of $P$. infestans with the regulation of the transition from biotrophic to necrotrophic stages $[3,31]$.

The RXLR effectors are almost always related to the biotrophic stage of the infection [3]. Therefore, the finding that a small ncRNA associated with the Avr2 family secreted RxLR effector was significantly upregulated at the necrotrophic stage was expected. The expression of the gene associated with this small ncRNA was validated using qRT-PCR. In this experiment, it was evident that this gene was only slightly upregulated during biotrophy and then became downregulated during both the transition from biotrophy to necrotrophy and the necrotrophic phases. This finding supports the idea that the small ncRNA associated with the gene may be one of the regulatory mechanisms affecting the expression of this gene, especially during necrotrophy. Furthermore, it supports the idea that RXLR effectors are mostly expressed during the biotrophic stage, as suggested by Zuluaga and collaborators (2016).

Similarly, elicitins and elicitin-like proteins are relevant effectors expressed at different time points of the infection cycle. Some are known to be highly expressed during the biotrophic phase (early in the infection), while others are highly expressed during the necrotrophic phase (at later time points in the infection cycle) [3]. In this study, we identified small ncRNAs associated with the elicitin-like proteins INF1 , INF-6, and INF-2A, which have been defined as important necrotrophic elicitors $[3,32]$ or as inducers of various degrees of the hypersensitive response or programmed cell death in plants [33]. Small ncRNAs associated with these elicitin-like proteins were significantly downregulated at every time point when compared to mycelia grown in media. Thus, the genes associated with these small ncRNAs are expected to be overexpressed throughout the disease cycle. However, the genes associated with INF1 and INF6 were evaluated by qRT-PCR and were found to be downregulated during all stages of the cycle when compared to the in vitro mycelia, which does not support the expected expression profile of the genes. This highlights the complexity of the disease progression gene regulation.

A wide variety of small ncRNAs associated with enzymatic processes were found to be differentially expressed across the majority of the comparisons made. Their up- and downregulation is highly likely to be involved in how the pathogen responds to host defenses. One example is the sporangia-induced phosphatidylinositol kinase, which, as its name suggests, is usually induced in response to sporangia production in $P$. infestans. In this case, the small ncRNA associated with the gene seems to be upregulated at the beginning of the infection when compared to the transition from the biotrophy to the necrotrophy stage and to the in vitro mycelia (Table 1). Therefore, the associated gene would be expected to be downregulated during the biotrophic stage when compared to necrotrophy and to the in vitro mycelia. This is consistent with the function of this gene because $P$. infestans mainly feeds on the host during the biotrophic phase [3]. Therefore, the production of sporangia is most likely to occur near the end of the necrotrophic stage when the pathogen needs to proliferate and disperse to other plants. The qRTPCR analyses showed that this gene is downregulated in all stages when compared to the in vitro mycelia 
(this was less evident in biotrophy and necrotrophy than in the transition between them). This suggests that the isolate had not sporulated in the specific moment of the necrotrophic stage that was tested and potentially needed more time to sporulate.

In a similar sense, we evaluated the peptidyl-prolyl cis-trans isomerase, an enzyme that accelerates protein folding and may be part of a downstream reaction in the infection process. Based on the differential expression of its associated small ncRNAs (Table 1), the peptidyl gene would be expected to be overexpressed during biotrophy. This is because its small ncRNA was downregulated when comparing $24 \mathrm{hpi}$ (biotrophy) to $48 \mathrm{hpi}$ (transition to necrotrophy). The qRT-PCR results support an overexpression of the peptidyl-prolyl cis-trans isomerase gene during biotrophy followed by a downregulation during the transition to necrotrophy when compared to the in vitro mycelia.

The nuclear LIM factor interactor-interacting protein spore-specific form gene was predicted to be downregulated during biotrophy when compared to the in vitro mycelia because the corresponding small ncRNA was upregulated when these two conditions were compared (see Table 1). However, this gene was found to be ubiquitously upregulated in all stages of the infection when compared to the in vitro mycelia based on the qRT-PCR assays. In theory, these types of proteins are zoosporogenesis induced, and they seem to perform a role of phosphatases when $P$. infestans is producing zoospores [34, 35]. Thus, they are expected to be upregulated at later stages of the infection when the zoospores are being produced. However, the pattern obtained does not clearly support the involvement of small ncRNAs in the regulation of the expression of this gene.

Berberine-like proteins are known to be involved in alkaloid biosynthesis and hydrogen peroxide production [36]. Hence, they are important virulence factors during plant infection and could also perform a protective role in Phytophthora against the plant defenses [36]. A Berberine-like protein gene would be expected to be downregulated when comparing its expression during necrotrophy with that of in vitro mycelia. This is because its corresponding small ncRNA was upregulated when comparing these two stages, which is consistent with its predicted defensive role. The small ncRNA was also upregulated when comparing biotrophy to the in vitro mycelia. This is also accurate if we consider that Meijer and collaborators (2014) discussed that, even though their data cannot shed extra light on the function of these proteins, the data can certainly support the idea that these proteins are expressed during hyphal growth, which can also be encountered during biotrophy to a lesser extent. The berberine-like protein gene was upregulated during biotrophy, which supports the idea that small ncRNAs may only partially regulate the expression of the genes involved in the infection processes.

Finally, small ncRNAs associated with ATP-binding cassette (ABC) transporters (also listed in Table 1) had variable expression. They were initially upregulated, then downregulated and then again upregulated, which concurs with a known pattern characteristic of the transition from biotrophy to necrotrophy. This is because of the response of $P$. infestans to host defenses. Since common host defenses include the production of reactive oxygen species (ROS) and $A B C$ transporters are involved in detoxification [3], it is 
reasonable to expect that their expression increases as the infection progresses towards the necrotrophic stage.

The differential expression found for these small ncRNAs indicates that the $A B C$ transporter genes are expected to present an initial downregulation during biotrophy, then an upregulation during the transition from biotrophy to necrotrophy and then a downregulation again during necrotrophy (Table 1). This behavior allows the pathogen to protect itself from possible plant host defenses. Interestingly, our qRTPCR experiments confirmed an initial downregulation of $A B C$ transporter \# 1 during biotrophy when compared to the in vitro mycelia. Subsequently, an increase in its expression to a level similar to that of the in vitro mycelia (at $48 \mathrm{hpi}$ ) and then an upregulation during necrotrophy were observed. Although this expression pattern is not exactly as expected, it is again partially supported by the role of the small ncRNAs as regulators of gene expression. As with the haustorium-specific membrane protein gene, $A B C$ transporter \#2 did not demonstrate differential expression in the qRT-PCR assays. Again, testing a larger number of ATP-binding transporters and specific haustorium membrane proteins might be necessary to understand their expression profiles with more precision.

Similar studies whose aim is to understand more about the biology of small ncRNAs in Phytophthora species have also included validation steps to evaluate if the expression of the genes is consistent with the expression predicted based on the expression profiles of the small ncRNAs. These studies usually focus on one or a few small ncRNAs or miRNAs and test their expression in a very detailed manner. Luan and collaborators (2016) evaluated a specific miRNA called MIR1918 and then validated its expression via qRT-PCR experiments. Moreover, Cui and collaborators (2014) evaluated the expression of four micro RNAs using a qRT-PCR-based approach. The first of these studies was able to prove that the miRNA seemed to have a negative effect on the expression of the associated gene [13], and the second was able to corroborate that the miRNA expression levels were as predicted by the bioinformatics analyses [14].

Jia and collaborators (2017) were interested in evaluating a broader amount of small ncRNAs. To do this, they performed transcriptomic analyses, mainly RNA-Seq, and searched for the presence or absence of the transcripts of genes associated to the small ncRNAs. This allowed them to test for the small ncRNAs' possible effect in a much more massive way. They also concluded that while 25-26 nt small ncRNAs seem to regulate gene silencing in Phytophthora spp., those small ncRNAs of $21 \mathrm{nt}$ do not seem to participate in this process or at least they do not seem to be the sole regulatory mechanism mediating it [8]. This could also explain why our results indicate a clear association between the small ncRNAs and their correspondent genes in some cases while in others it is not that clear. Also, it is important to consider that small ncRNAs may also be acting indirectly on the expression of genes involved in the process of infection by the regulation of the expression of other genes whose resultant proteins may promote or inhibit the infection's progression [37].

Interestingly, several differentially expressed small ncRNAs were associated with genes encoding putative uncharacterized proteins. Future processing of these sequences, possibly by means of a conserved domain analysis search, could be implemented to further investigate their function. As expected, most of 
the small ncRNAs found to be significantly differentially expressed were identified from comparisons that involved data from the mycelia grown in culture media. This is most likely because, in this case, $P$. infestans is not exposed to the host, and thus, it is expected that its regulatory processes would be very different from those in any of the other data sets tested.

The presence of some tRNAs, pseudo tRNAs, and one rRNA among the small ncRNAs that showed statistically significant differential expression was not expected due to the removal of these types of RNAs in the implemented pipeline (blue bar in Figure 1). However, the tRNA and rRNA repositories to which the reads were aligned were the ones that performed best among several that were tested. Furthermore, it is important to consider that the genome of $P$. infestans is highly repetitive [11] and therefore contains regions that might not be aligning completely, thus hindering the removal of these contaminant sequences.

\section{Conclusions}

The expression of small ncRNAs in $P$. infestans seems to be correlated with the expression of effector genes in this pathogen during its hemibiotrophic life cycle as well as with other molecular regulatory processes that may also take place during the biotrophic, transition to necrotrophic and necrotrophic phases. This correlation should not necessarily be confused with strict causality, and the statistically significant differential expression of these small ncRNAs could be either a cause or a consequence for this correlation, which must be further investigated in future studies. Furthermore, small ncRNAs could be involved only partly in regulating the expression of these genes or may also have an indirect role in it. The pipeline presented here is a suitable option for performing these types of analyses using $P$. infestans small RNA-seq data sets since customized pipelines are not available for this purpose.

\section{Methods}

\section{Implementation of a bioinformatic pipeline to analyze small ncRNA data}

\section{Acquisition of raw RNA-seq data}

To test the proposed pipeline (Figure 1) and identify possible small ncRNAs regulating the transition from biotrophy to necrotrophy in $P$. infestans, RNA-seq data sets were extensively searched in NCBI databases (mainly GEO DataSets [19] and SRA (http://www.ncbi.nlm.nih.gov/sra), specialized RNA-seq databases (such as RNAcentral (RNAcentral Consortium, 2014), and available literature (gray bar in Figure 1). However, only one data set initially published by Asman and collaborators (2014) [20] (GEO accession number: GSE63292) included all the required characteristics. The criteria for inclusion were i) data only from $P$. infestans, ii) data obtained using sequencing libraries intended for small RNAs (those using hexamers or a commercially available kit) but not from mRNA selection methods such as polyadenylation filters, and iii) data that included small RNA extractions at various life cycle stages 
allowing a much more precise analysis of the transition from biotrophy to necrotrophy. Briefly, the methodology implemented to obtain the data from the selected study involved the inoculation of leaves from Solanum tuberosum infected with $P$. infestans and the subsequent extraction and sequencing of small ncRNAsat 24,48 , and 72 hours post inoculation (hpi). The authors also included data from mycelia grown in culture media where the pathogen is not exposed to the plant as a negative control. Under the conditions in which the inoculations were performed and for the specific isolate that was analyzed $(P$. infestans isolate 88069), $24 \mathrm{hpi}$ was the biotrophic stage, $48 \mathrm{hpi}$ was considered to be the transition from biotrophy to necrotrophy, and 72 hpi was the necrotrophic stage [20].

\section{RNA-seq data processing}

The selected data set was processed and optimized for the RNA-seq data analysis. This was a two-step process: data quality trimming and ncRNA selection (orange and blue bars in Figure 1). In the first step, the quality of the data was assessed in order to identify and subsequently discard low-quality sections. Fastq-dump (SRA toolkit) and FastQC were used to convert the SRA data files to FASTQ format and assess their initial quality (http://www.bioinformatics.babraham.ac.uk/projects/fastqc). The reads were of very good quality (over 40 Phred quality score) in all bp except for the last one, which was removed using cutadapt [21]. This process was performed only on the reads that contained adaptor sequences. Subsequently, adaptor sequences were removed, and the reads were selected by size using cutadapt in order to keep only those ranging between $18-30 \mathrm{bp}$. This was done to eliminate RNA sequences that had not been processed adequately $[9,10,20]$.

In the second step, rRNAs and tRNAs (housekeeping RNAs) [9] were eliminated from the remaining reads in order to limit the analyses only to regulatory RNA species. Known rRNA and tRNA sequences for $P$. infestans were downloaded from Ensembl Protists using the BioMart tool (http://protists.ensembl.org/). Reads were first aligned to the rRNA sequences using Bowtie 1 [22], and the non-aligned reads were kept (rRNAs were removed). The same process was performed to remove tRNAs. Both alignments were performed with Bowtie by reporting the first valid alignment $(-k 1)$ and allowing 0 mismatches in the alignment. Finally, the resulting reads were aligned to the $S$. tuberosum genome (from NCBI) using the same alignment parameters. At this point, data sets were completely free from interfering elements and ready for further analysis.

\section{RNA-seq data analysis}

After processing the small RNA-seq data, the analysis was initiated with the alignment of small RNA reads to the T30-4 P. infestans genome from the Broad Institute (available at http://protists.ensembl.org/index.html) using Bowtie1 [9, 10, 20, 23]. This alignment was performed with the same parameters mentioned before (-v 0 and $-\mathrm{k} 1)$. After this, the GTF file with the annotated $P$. infestans was obtained from Ensembl Protists. This type of file contains the current annotation of the $P$. infestans genome. This file and the SAM file that resulted from the alignment were then used to generate count tables that indicated how many times each small ncRNA was aligned in each data set analysis. For example, the small ncRNA mapping to the gene "abc" was aligned "xxx" times in the $24 \mathrm{hpi}$ data set. This 
was done using HTSeq [24] with the htseq-count feature and the "union" intersection type, meaning that read identity is cataloged as "ambiguous" only when it aligns to a position where two genes overlap.

HTSeq count tables were then used as an input for DESeq2 [25] to determine small ncRNAs that were differentially expressed when comparing each data set to the others. DESeq2 normalizes the input count tables by the Relative Log Expression (RLE) method [26]. This analysis was performed online in Galaxy [27]. The factor specified for the analysis was "hpi", and it had four levels: $24,48,72$, and the mycelia grown in culture mediaall of these steps are represented in the purple bar in Figure 1). The threshold for the $p$-values associated with the $\log _{2}$ fold change $(F C)$ of each small ncRNA was fixed at 0.05 . Finally, each small ncRNA found to be significantly differentially expressed was annotated using the program Blast2GO, and the small ncRNAs that were successfully annotated to specific genes were classified into nine categories based on their molecular functions or cellular processes (Tables 1 and A1).

\section{Generation of qRT-PCR profiles for gene expression validation}

\section{Tissue generation and collection}

To evaluate whether the predicted small ncRNAs (Table A1) may regulate the expression of their corresponding genes during the transition from biotrophy to necrotrophy in P. infestans, the expression of the genes associated with ten of the small ncRNAs was evaluated using qRT-PCR. Instead of directly validating the expression of small ncRNAs, we decided to validate and study the expression of the genes that were predicted by our bioinformatic analyses to be associated with these small ncRNAs. In this way we were able to study a greater number of potential associations between small ncRNAs and genes, instead of focusing in just a few of the predicted small ncRNAs. This approximation is coherent to what was done by Jia and collaborators (2017), who predicted both small ncRNAS and their associated genes using RNA-seq and then validated their results using qPCR to study gene expression levels. These ten genes could potentially be associated with each phase (biotrophic, transition to necrotrophic, or necrotrophic phase) based on what is currently known about the genes mediating the life cycle of $P$. infestans $[3,4]$ and they represent all the functional categories that were identified in the bioinformatic analyses.

To this end, Solanum tuberosum leaves were inoculated in vitro with the $P$. infestans isolate RC1\#10 (of the EC-1 clonal lineage). Solanum tuberosum plants of the Diacol Capiro variety were grown for one and a half months to obtain leaves of an adequate age for in vitro inoculation. Isolate RC1\#10 was grown in pea agar for seven days and kept at $20^{\circ} \mathrm{C}$.

After one week of incubation, Petri dishes containing RC1\#10 mycelia were scraped to obtain a $1 \mathrm{~mL}$ sporangial suspension. This solution was diluted to a concentration of $1 \times 10^{3}$ sporangia $\mathrm{mL}^{-1}$. Two $10-\mu \mathrm{L}$ drops of the sporangial suspension were placed on each side of the vein of the leaf placed abaxial side up. The leaves were maintained in moist chambers until symptoms developed (three leaflets per petri dish). Subsequently, two transfers to healthy leaflets were performed by immersing the diseased leaves 
into distilled water, adjusting the solution to $1 \times 10^{3}$ sporangia per $\mathrm{mL}$, if necessary, and repeating the previously mentioned inoculation process. This was done in order to obtain sporangia that came directly from the leaf and not from the medium in the Petri dishes.

Plant tissue was sampled $12 \mathrm{hpi}$ (biotrophic stage), $48 \mathrm{hpi}$ (transition from biotrophy to necrotrophy), and $96 \mathrm{hpi}$ (necrotrophic stage). Time points for each phase of the infection process were determined specifically for this isolate and this potato variety under the specific conditions assessed. The area where each droplet was placed was cut out and frozen at $-80^{\circ} \mathrm{C}$. Sporangia from mycelia growing in pea agar were also collected as a control and stored at $-80^{\circ} \mathrm{C}$.

\section{RNA Extraction and cDNA conversion}

Total RNA was extracted from the infected plant tissue at each stage of the disease and the in vitro control using RNeasy Plant Mini Kit (QIAGEN, Hilden, Germany) following the manufacturer's instructions. The RNA quality was assessed in an agarose gel, and the quantity was tested with a NanoDrop 1000 Spectrophotometer (Thermo Fisher Scientific, Waltham, MA, USA). Subsequently, the extracted RNA was converted to cDNA using RevertAid H Minus First Strand cDNA Synthesis Kit (ThermoFisher) following the manufacturer's instructions.

\section{qRT-PCR profiles}

Real-time reverse transcription PCR (qRT-PCR) was performed to obtain an expression profile of the genes associated with 10 of the small ncRNAs predicted to be differentially expressed between each infection stage and the control mycelia or between the infection stages. The ten genes are summarized in Table 2 along with the stages in which they were up- or downregulated. Total transcript levels were determined using the Luna Universal qPCR Master Mix (2X) (New England BioLabs, Ipswich, MA, USA), following the manufacturers' protocol. Gene primers were designed exon to exon using Primer-BLAST software [28] taking into account all recommendations provided by the Luna Universal qPCR Master Mix (2X) protocol (New England BioLabs, Ipswich, MA, USA) (Table 3). The P. infestans 40S ribosomal protein S3A (PITG_04425) was used as a constitutively expressed endogenous control [29]. All genes were assayed in triplicate in MicroAmp ${ }^{\text {TM }}$ Fast Optical 96-Well Reaction Plates (Applied Biosystems, Foster City, CA, USA), and three biological replicates of each treatment were performed. A control lacking template was included in the plates, and a control lacking reverse transcriptase was included for all extracted RNA samples. RNA from $P$. infestans mycelia grown in vitro was used as the calibrator. The results were analyzed with the 7500 Fast Real-Time PCR System (Applied Biosystems, Foster City, CA, USA), and relative expression was calculated using the REST software [30].

\section{List Of Abbreviations}

ncRNAs: Noncoding RNAs

tRNAs: Transfer RNAs

Page 12/23 
rRNAs: Ribosomal RNAs

hpi: Hours post inoculation

bp: Base pair

$n t:$ Nucleotides

\section{Declarations}

\section{Ethics approval and consent to participate}

Not applicable.

\section{Consent for publication}

Not applicable.

\section{Availability of data and materials}

The data sets generated and/or analyzed in the current study are available in the GitHub repository https://github.com/jgonzalez10/Small-ncRNAs-pipeline

\section{Competing interests}

The authors declare that they have no competing interests.

\section{Funding}

No funding was received for this study.

\section{Author contributions}

JG-T developed and implemented the proposed pipeline, analyzed the results, performed all the inoculation steps, the qRT-PCR experiments, and wrote the manuscript. AR-J helped perform the qRT-PCR experiments and the inoculation steps. LMF implemented the pipeline and contributed significantly to the bioinformatic analyses. LNG provided general guidance for the development of the study, specifically for the bioinformatic pipeline implementation. GD provided general guidance for the development of the study and made substantial contributions to writing and editing the manuscript. SR provided continuous and profound guidance on the development of the manuscript and analyzed the results and was also a major contributor in writing the manuscript. All authors read and approved the final manuscript. 


\section{Acknowledgments}

We thank Jose A. Vargas for his valuable advice during the development of this work.

\section{References}

1. Schoina C, Govers F. The Oomycete Phytophthora infestans, the Irish Potato Famine Pathogen. In: Lugtenberg B, editor. Principles of Plant Microbe Interactions. Switzerland: Springer International Publishing. 2015;371-78.

2. Fry W. Phytophthora infestans: The plant (and R gene) destroyer. Molecular Plant Pathology. 2008;9:385-402.

3. Zuluaga AP, Vega-Arreguín JC, Fei Z, Ponnala L, Lee SJ, Matas AJ, et al. Transcriptional dynamics of Phytophthora infestans during sequential stages of hemibiotrophic infection of tomato. Molecular Plant Pathology. 2016;17:29-41.

4. Lee SJ, Rose JKC. Mediation of the transition from biotrophy to necrotrophy in hemibiotrophic plant pathogens by secreted effector proteins. Plant Signaling \& Behavior. 2010;5:769-72.

5. Gijzen M, Ishmael C, Shrestha, SD. Epigenetic control of effectors in plant pathogens. Frontiers in Plant Science. 2014;5:1-5.

6. Kasuga T, Gijzen M. Epigenetics and the evolution of virulence. Trends Microbiol. 2013;21:575-82.

7. Moazed D. Small RNAs in transcriptional gene silencing and genome defense. Nature. 2009;457: 413-20.

8. Jia, J., Lu, W., Zhong, C., Zhou, R., Xu, J., Liu, W., ... \& Shan, W. The 25-26 nt small RNAs in Phytophthora parasitica are associated with efficient silencing of homologous endogenous genes. Frontiers in microbiology. 2017;8:773.

9. Vetukuri RR, Åsman AK, Tellgren-Roth C, Jahan SN, Reimegård J, Fogelqvist J, et al. Evidence for Small RNAs Homologous to Effector-Encoding Genes and Transposable Elements in the Oomycete Phytophthora infestans. PLoS ONE. 2012;7:e51399.

10. Fahlgren N, Bollmann SR, Kasschau KD, Cuperus JT, Press CM, Sullivan CM, et al. Phytophthora Have Distinct Endogenous Small RNA Populations That Include Short Interfering and microRNAs. PLoS ONE. 2013;8:e77181.

11. Haas BJ, Kamoun S, Zody MC, Jiang RHY, Handsaker RE, Cano LM, et al. Genome sequence and analysis of the Irish potato famine pathogen Phytophthora infestans. Nature. 2009;461:393-8.

12. Wang, Q., Li, T., Xu, K., Zhang, W., Wang, X., Quan, J., ... \& Shan, W. The tRNA-derived small RNAs regulate gene expression through triggering sequence-specific degradation of target transcripts in the oomycete pathogen Phytophthora sojae. Frontiers in plant science. 2016;7: 1938.

13. Luan, Y., Cui, J., Wang, W., \& Meng, J. MiR1918 enhances tomato sensitivity to Phytophthora infestans infection. Scientific reports. 2016;6:35858. 
14. Cui, J., Luan, Y., Wang, W., \& Zhai, J. Prediction and validation of potential pathogenic microRNAs involved in Phytophthora infestans infection. Molecular biology reports. 2014;41(3):1879-1889.

15. Bollmann, Stephanie R., et al. Diverse evolutionary trajectories for small RNA biogenesis genes in the oomycete genus Phytophthora. Frontiers in plant science. 2016;7:284.

16. Rueda A, Barturen G, Lebrón R, Gómez-Martín C, Alganza Á, Oliver JL, et al. sRNAtoolbox: an integrated collection of small RNA research tools. Nucleic Acids Res. 2015;43:W467-73.

17. Giurato G, De Filippo MR, Rinaldi A, Hashim A, Nassa G, Ravo M, et al. iMir: An integrated pipeline for high-throughput analysis of small non-coding RNA data obtained by small RNA-Seq. BMC Bioinformatics. 2013;14:1-9.

18. Gupta V, Markmann K, Pedersen CN, Stougaard J, Andersen SU. shortran: a pipeline for small RNAseq data analysis. Bioinformatics. 2012;28:2698-700.

19. Edgar R, Domrachev M, Lash AE. Gene Expression Omnibus: NCBI gene expression and hybridization array data repository. Nucleic Acids Res. 2002;30:207-10.

20. Åsman AKM, Vetukuri R, Jahan SN, Fogelqvist J, Corcoran P, Avrova AO, et al. Fragmentation of tRNA in Phytophthora infestans asexual life cycle stages and during host plant infection. BMC Microbiology. 2014;14:1-12.

21. Martin M. Cutadapt removes adapter sequences from high-throughput sequencing reads. EMBnet.journal. 2011;17:10-2.

22. Langmead B, Trapnell C, Pop M, Salzberg, SL. Ultrafast and memory-efficient alignment of short DNA sequences to the human genome. Genome Biology. 2009;10:R25.

23. Åsman AKM, Fogelqvist J, Vetukuri R, Dixelius, C. Phytophthora infestans Argonaute 1 binds microRNA and small RNAs from effector genes and transposable elements. New Phytologist. 2016;211:993-1007.

24. Anders S, Pyl PT, Huber W. HTSeq-a Python framework to work with high-throughput sequencing data. Bioinformatics. 2015;31:166-9.

25. Love MI, Huber W, Anders S. Moderated estimation of fold change and dispersion for RNA-seq data with DESeq2. Genome Biology. 2014;15:1-21.

26. Reddy, R. A comparison of methods: normalizing high-throughput RNA sequencing data. bioRxiv. 2015;026062.

27. Afgan E, Baker D, van den Beek M, Blankenberg D, Bouvier D, Čech M, et al. The Galaxy platform for accessible, reproducible and collaborative biomedical analyses: 2016 update. Nucleic Acids Res. 2016;44:W3-10.

28. Ye, J., Coulouris, G., Zaretskaya, I., Cutcutache, I., Rozen, S., \& Madden, T. L. Primer-BLAST: a tool to design target-specific primers for polymerase chain reaction. BMC bioinformatics. 2012;13(1):134.

29. Yan, H. Z., \& Liou, R. F. Selection of internal control genes for real-time quantitative RT-PCR assays in the oomycete plant pathogen Phytophthora parasitica. Fungal Genetics and Biology. 2006;43(6):430-438. 
30. Pfaffl, M. W., Horgan, G. W., \& Dempfle, L. Relative expression software tool (REST@) for group-wise comparison and statistical analysis of relative expression results in real-time PCR. Nucleic acids research. 2002;30(9):e36-e36.

31. Stam R, Jupe J, Howden AJ, Morris JA, Boevink PC, Hedley PE, et al. Identification and characterization CRN effectors in Phytophthora capsici shows modularity and functional diversity. Plos one. 2013;8:e59517.

32. Kanneganti TD, Huitema E, Cakir C, Kamoun S. Synergistic Interactions of the Plant Cell Death Pathways Induced by Phytophthora infestans Nep1-Like Protein PiNPP1.1 and INF1 Elicitin. MPMI. 2006;19:854-63.

33. Kamoun S. Nonhost resistance to Phytophthora: novel prospects for a classical problem. Current opinion in plant biology. 2001;4:295-300.

34. Tani, S., \& Judelson, H. Activation of zoosporogenesis-specific genes in Phytophthora infestans involves a 7-nucleotide promoter motif and cold-induced membrane rigidity. Eukaryotic cell. 2006;5(4):745-752.

35. Judelson, H. S., \& Tani, S. Transgene-induced silencing of the zoosporogenesis-specific NIFC gene cluster of Phytophthora infestans involves chromatin alterations. Eukaryotic cell. 2007;6(7):12001209.

36. Meijer, H. J., Mancuso, F. M., Espadas, G., Seidl, M. F., Chiva, C., Govers, F., \& Sabido, E. Profiling the secretome and extracellular proteome of the potato late blight pathogen Phytophthora infestans. Molecular \& Cellular Proteomics. 2014;13(8):2101-2113.

37. Peschansky, V. J., \& Wahlestedt, C. Non-coding RNAs as direct and indirect modulators of epigenetic regulation. Epigenetics. 2014;9(1):3-12.

38. Maze I, Shen L, Zhang B, Garcia BA, Shao N, Mitchell A, et al. Analytical tools and current challenges in the modern era of neuroepigenomics. Nature Neuroscience. 2014;17:1476-90.

\section{Tables}

Table 1. Small ncRNAs were significantly upregulated and downregulated in Phytophthora infestans in infected potato leaves. These ncRNAs divided in nine categories according to their annotation. Comparisons of the ncRNAs expression were made between each of the time points $(24,48$, and 72 hours post inoculation) as well as with mycelia grown in culture media. The numbers in the first column of each comparison between data sets indicate the number of up- and/or downregulated small ncRNAs in each category. Some ncRNAs categories have two rows when some ncRNAs in this particular category were up and others downregulated. 


\begin{tabular}{|c|c|c|c|c|c|c|c|c|c|c|c|}
\hline \multirow[t]{2}{*}{ Small ncRNAs categories } & \multicolumn{11}{|c|}{ Comparisons made between data sets } \\
\hline & \multicolumn{2}{|c|}{$\begin{array}{l}24 \text { vs. } \\
48\end{array}$} & \multicolumn{2}{|c|}{$\begin{array}{l}24 \text { vs. } \\
72\end{array}$} & $\begin{array}{l}48 \text { vs. } \\
72\end{array}$ & \multicolumn{2}{|c|}{$\begin{array}{c}24 \text { vs. } \\
\text { mycelia }\end{array}$} & \multicolumn{2}{|c|}{$\begin{array}{c}48 \text { vs. } \\
\text { mycelia }\end{array}$} & \multicolumn{2}{|c|}{$\begin{array}{c}72 \text { vs. } \\
\text { mycelia }\end{array}$} \\
\hline Small nucleolar RNA & 1 & - & 1 & - & & 4 & - & & & & \\
\hline \multirow[t]{2}{*}{ Spliceosomal RNA } & & & & & & 1 & - & 6 & - & 1 & - \\
\hline & & & & & & & & & & & \\
\hline \multirow{2}{*}{$\begin{array}{c}\text { Eukaryotic type signal recognition } \\
\text { particle RNA }\end{array}$} & & & & & & 1 & - & & & 2 & - \\
\hline & & & & & & & & & & & \\
\hline \multirow[t]{2}{*}{ Elicitin and elictin-like proteins } & & & & & & 3 & - & 3 & - & 2 & - \\
\hline & & & & & & & & 3 & - & 14 & 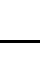 \\
\hline Crinklers and crinkler-like proteins & & & & & & & & & & & \\
\hline \multirow[t]{2}{*}{ Avr2 family secreted RxLR effector } & & & & & & & & & & 1 & + \\
\hline & & & & & & & & & & & \\
\hline \multirow[t]{2}{*}{ Enzymatic processes } & 1 & - & 1 & + & & 10 & - & 10 & - & 5 & - \\
\hline & & & & & & 6 & + & 1 & + & 4 & + \\
\hline \multirow{2}{*}{$\begin{array}{c}\text { Transporters and transmembrane } \\
\text { proteins }\end{array}$} & & & & & & 2 & - & 4 & - & 2 & - \\
\hline & & & & & & 2 & + & & & 2 & + \\
\hline \multirow[t]{2}{*}{ DNA binding or binding sites } & & & & & & 4 & - & 4 & - & 1 & - \\
\hline & & & & & & 1 & + & & & 1 & + \\
\hline
\end{tabular}

+: upregulated small ncRNAs, -: downregulated small ncRNAs.

Table 2. Genes associated with small ncRNAs that were significantly differentially expressed during different stages (biotrophic, transition to necrotrophic, and necrotrophic) of the infection cycle of Phytophthora infestans. qRT-PCR was performed to confirm that the genes were significantly differentially expressed during the different infection stages. 


\begin{tabular}{|c|c|c|c|c|}
\hline $\begin{array}{c}\text { Genes to which small ncRNAs } \\
\text { aligned to }\end{array}$ & Gene ID & Category & $\begin{array}{l}\text { Differential } \\
\text { expression of } \\
\text { the small } \\
\text { ncRNAs }\end{array}$ & $\begin{array}{c}\text { Data set in which the } \\
\text { differences in } \\
\text { expression occur }\end{array}$ \\
\hline
\end{tabular}

Avr2 family secreted RxLR PITG 22870 Avr2 family Upregulated 72 hpi vs, mycelium

effector peptide protein

Elicitin (INF1)

Elicitin-like INF6

Sporangia

phosphatidyl inositol kinase

induced PITG_20221 Enzymatic

processes

\section{Peptidyl-prolyl}

cis-trans

isomerase

Nuclear LIM factor interactor-

interacting protein spore-

specific form

Berberine-like protein

PITG_12556

effector processes processes like proteins

PITG_12551 Elicitin and elicitinlike proteins

RxLR

Downregulated

(1)

48 hpi vs. mycelium grown in culture

72 hpi vs. mycelium grown in culture 24 hpi vs. mycelium grown in culture

48 hpi vs. mycelium grown in culture

72 hpi vs. mycelium grown in culture 24 hpi vs. 72 hpi

24 hpi vs. mycelium grown in culture Downregulated $24 \mathrm{hpi}$ vs. $48 \mathrm{hpi}$

PITG_11241 Enzymatic Upregulated

PITG_02930 Enzymatic Upregulated processes/DNA binding or binding sites

ATP-binding Cassette (ABC) PITG_04289 Transporters and Downregulated Superfamily

ATP-binding Cassette (ABC) PITG_13576 Superfamily $\begin{aligned} & \text { Cassette (ABC) PITG_04289 } \text { Transporters } \\ & \text { transmembrane }\end{aligned}$ proteins

Transporters and Upregulated transmembrane
24 hpi vs. mycelium grown in culture 
proteins

72 hpi vs. mycelium grown in culture

Haustorium-specific membrane PITG_00375 Transporters and Upregulated protein
72 hpi vs. mycelium grown in culture transmembrane

proteins

Table 3. Exon to exon primers designed for the qRT-PCR-based assays of genes associated with significantly differentially expressed small ncRNAs. 


\begin{tabular}{|c|c|c|c|}
\hline Gene ID & Name & Forward primer & Reverse primer \\
\hline PITG_04425 & 40S Ribosomal protein S3A & CTGTCCATCCAAGCGCTACT & TTGAGGAACTGCTCGTGGTC \\
\hline PITG_22870 & $\begin{array}{l}\text { Avr2 family secreted } \mathrm{RxLR} \\
\text { effector peptide protein }\end{array}$ & GCCGTGTCGATAATGGGGAA & TCGCCTTTACCGCAGCTTTA \\
\hline PITG_12551 & Elicitin (INF1) & TTCTCGTCTACGTGTGCGTC & ACGCGGAGAACAAAGCCTAA \\
\hline PITG_12556 & Elicitin-like INF6 & CTCCACTTTCGAGACGCAGT & GAAGGAGCAGATGTAGGGGC \\
\hline PITG_20221 & $\begin{array}{l}\text { Sporangia induced } \\
\text { phosphatidylinositol kinase }\end{array}$ & CGCTCCGGTATCCCATACAC & GAAAAACTCGTTGGGTGGGC \\
\hline PITG_03856 & $\begin{array}{l}\text { Peptidyl-prolyl } \\
\text { isomerase }\end{array}$ & TCCTGCTAACGCAACACTGA & CGATCTTCTCCTCAGCGTCC \\
\hline PITG_11241 & $\begin{array}{l}\text { Nuclear LIM factor interactor- } \\
\text { interacting protein spore- } \\
\text { specific form }\end{array}$ & AAGACGTGGAAGATGTGCGT & AAGCAAGAGCTCATCGGACG \\
\hline PITG_02930 & Berberine-like protein & GTTGAGACCTCGCTACCCAC & CGCATTTCAGAGTGGCAGAA \\
\hline PITG_04289 & $\begin{array}{l}\text { ATP-binding cassette (ABC) } \\
\text { superfamily }\end{array}$ & CCGTCСТCTCATCTGTACGC & CATGTACTGCGGACACTGGT \\
\hline PITG_13576 & $\begin{array}{l}\text { ATP-binding cassette (ABC) } \\
\text { superfamily }\end{array}$ & TCTGCTCTGATGCCCAACG & TTGTAACCGGCCTTCATCGAC \\
\hline PITG_00375 & $\begin{array}{l}\text { Haustorium-specific membrane } \\
\text { protein }\end{array}$ & GGGTATCTTCGGTACGCTGG & TCCGTAGTCGGGATCAAGGT \\
\hline
\end{tabular}

\section{Figures}




\section{Raw RNAseq data gathering}

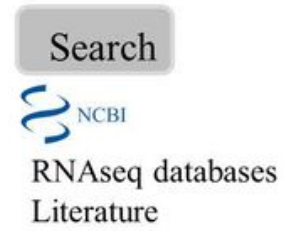

Selection
Species
Library construction
Life cycle (hpi)

Final data set

\section{Quality control of raw sequencing reads}

\section{Select}

- Only sequences with adaptors.

\section{Trim}

- bp with low quality.

- Adaptors.

- 18-30 bp size selection.

\section{Filtered RNAseq data}

\section{RNAseq data processing}

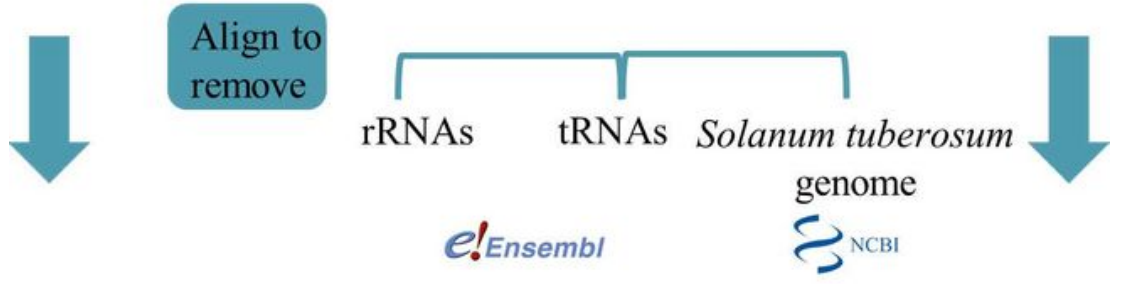

Processed RNAseq data

RNAseq data analysis

\section{Align}

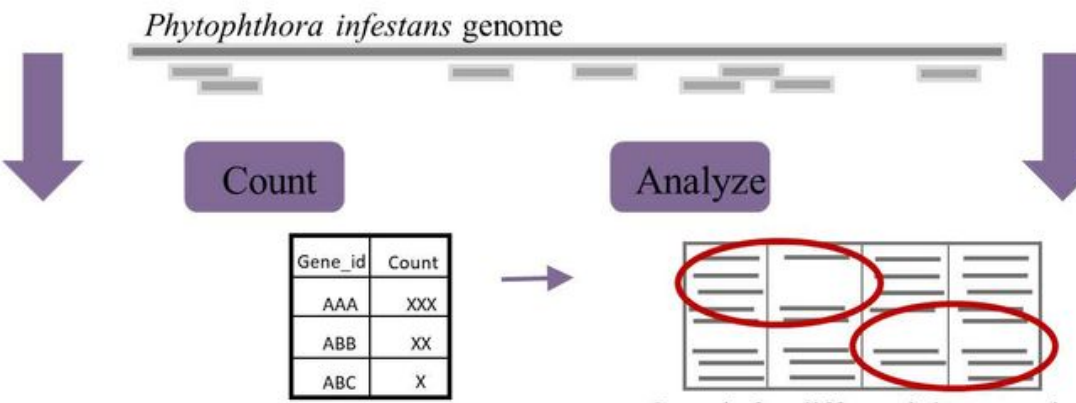

Search for differential expression.

\section{Pipeline results}

\section{Figure 1}

Methodological pipeline for the detection of small ncRNAs and analysis of small RNA-seq data. Adapted from [38]. 


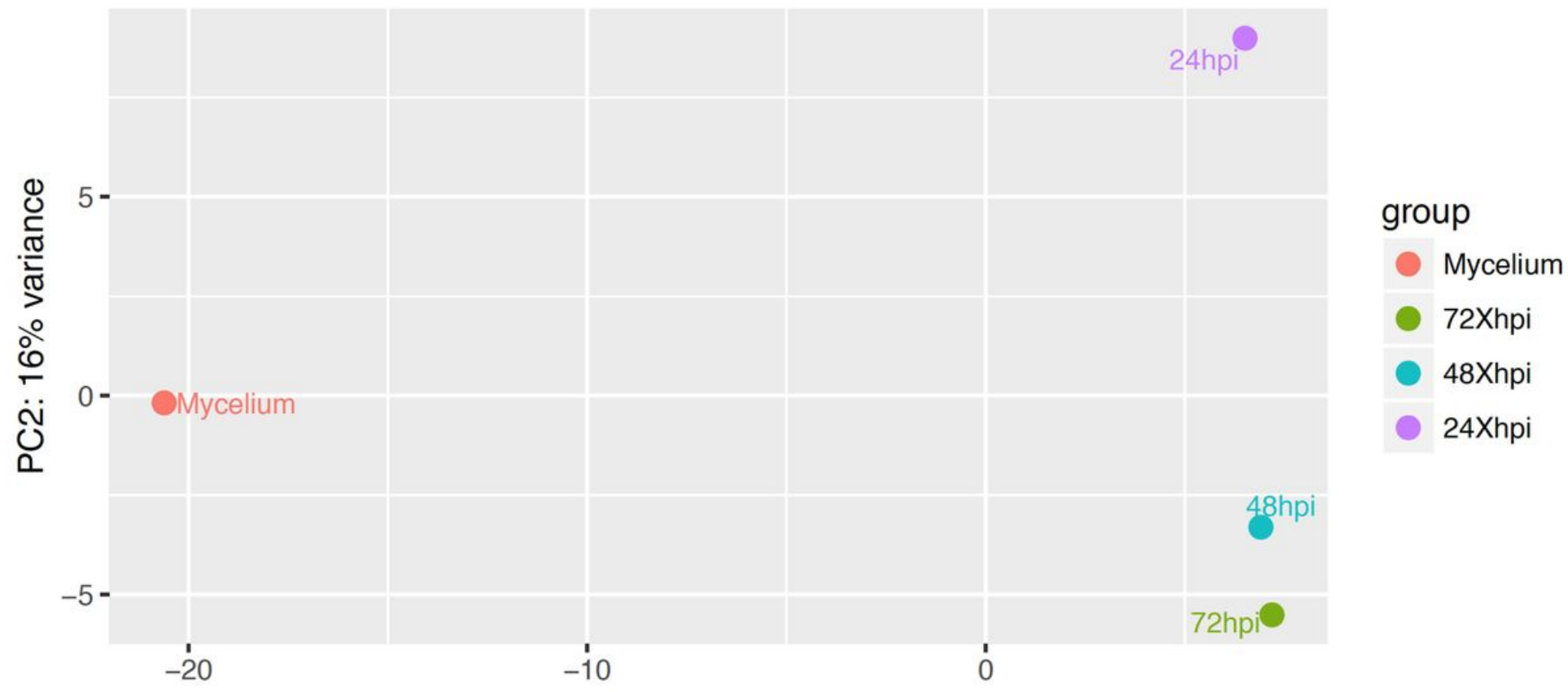

PC1: $73 \%$ variance

\section{Figure 2}

Principal components analysis (PCA) of the small ncRNAs belonging to the four analyzed data sets (24, 48 , and 72 hours post inoculation and mycelia grown in culture media) representing sample-to-sample distances.

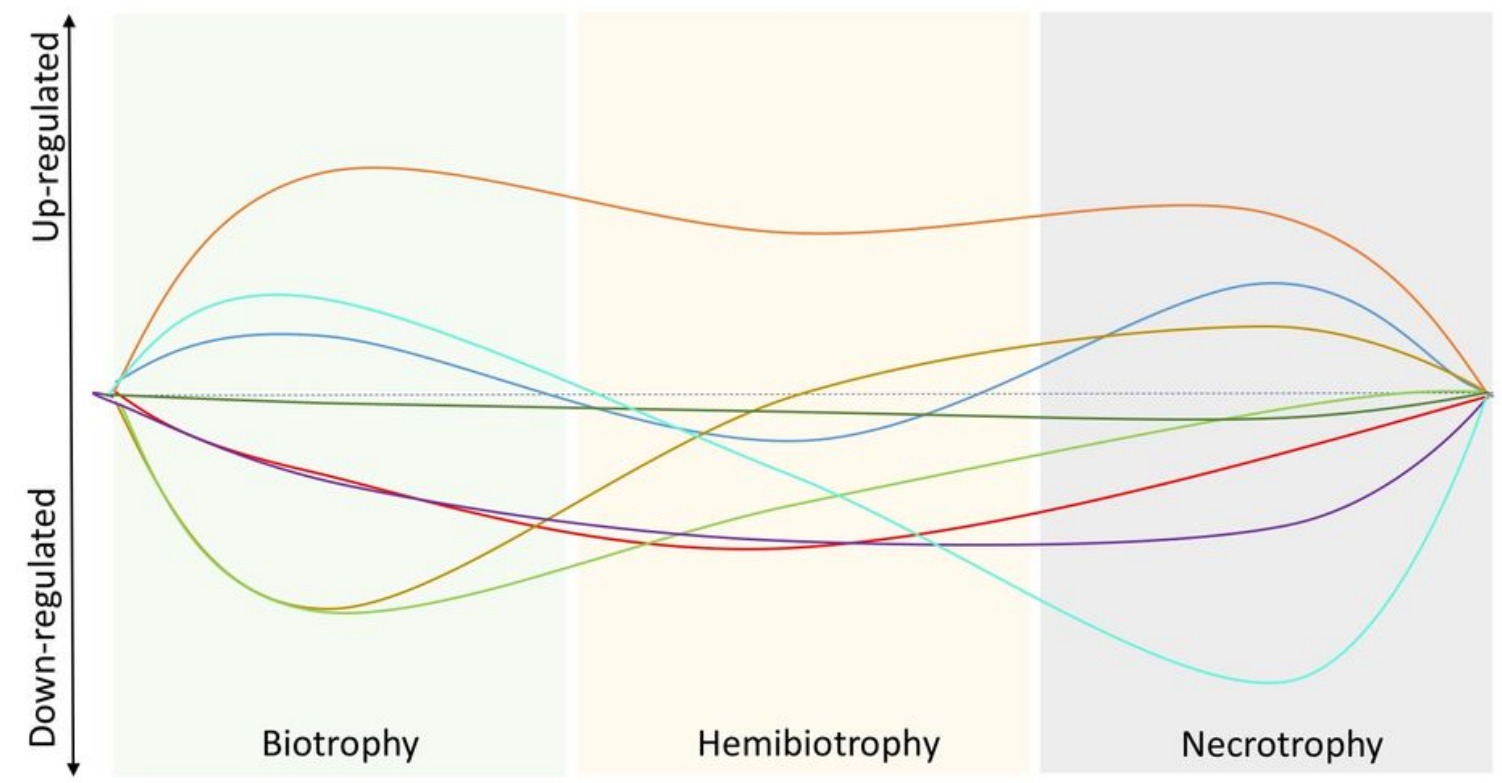

Peptidyl Sporkinase ABC\#1 INF1 INF6 SporeSpecific RXLR

Figure 3 
Expression levels of 10 genes selected due to their association with small ncRNAs expressed significantly differently between biotrophy, hemibiotrophy and necrotrophy, assessed by qRT-PCR. Each colored box represents one stage of the infection: biotrophy, the transition from biotrophy to necrotrophy and necrotrophy. Furthermore, each colored line represents each of the genes that were evaluated (see legend). Baseline gene expression is calculated in the in vitro mycelia, which is represented by the dotted line. Genes above this line were upregulated, while those below the line were downregulated when compared to the in vitro mycelia.

\section{Supplementary Files}

This is a list of supplementary files associated with this preprint. Click to download.

- supplement1.docx

- supplement2.docx 